\title{
Security Enhancement in Ad Hoc Network with ID-Based Cryptosystem
}

\author{
Jumin Song, Heeyoul Kim, Soojin Lee, H. Yoon \\ Computer Science Division \\ Korea Advanced Institute of Science and Technology \\ Republic of Korea \\ \{jmsong, hykim, sjlee, hyoon\}@camars.kaist.ac.kr
}

\begin{abstract}
As ad hoc network has advanced, the importance of security increases more and more. But it suffers from the restriction that ad hoc network does not have the established infrastructure. In this paper we propose a security-enhanced model with ID-Based cryptosystem which removes the necessity for any infrastructure and provides sound authentication and a more secure and concrete routing protocol with aggregate signature. ID-Based aggregate signature scheme, which is also proposed in this paper, removes the necessity for certificate transmission and verification. And it reduces communication cost significantly because our aggregate scheme compresses many signatures into only one signature keeping the same security level as existing signature schemes.
\end{abstract}

\section{INTRODUCTION}

Ad hoc network is composed of mobile nodes that carry out networking functions such as packet forwarding, routing, and network management. Ad hoc network has some different characteristics from the existing wired network. First of all, ad hoc network does not have the established infrastructure. And all available nodes in ad hoc network have to carry out networking functions while only dedicated nodes like routers support networking functions in the wired network. Due to these characteristics, ad hoc network is especially exposed to security threats. Therefore security in ad hoc network is an essential component for basic networking functions [8].

As ad hoc network has advanced in knowledge for the last years, security for ad hoc network becomes important more and more. Ad hoc network security research focuses on secure routing protocol, because routing protocol is a hot issue in ad hoc network. However, almost all the proposed secure routing protocols neglect the important problem in ad hoc network: authentication and key management.

Secure routing protocols such as SRP, Ariadne, ARAN, and SEAD [9], [6], [12], [5] all assume that two nodes of the network share the pre-existing symmetric key or public key infrastructure like a certificate authority exists. But these assumptions are not suitable for the characteristics of ad hoc network and raise many problems. In the symmetric key scheme [6], [5], it must have secure channel or pre-existing secure information to exchange the symmetric key between two nodes of the network, which raises a scalability problem. Also this scheme can not authenticate each node in network.
In the public key scheme [12], [14], each node has a pair of public key and private key. As opposed to the characteristics of ad hoc network, it needs the established infrastructure to distribute, manage, and revoke public keys.

The objective of this paper is to propose a comprehensive solution based on ID-based cryptosystem. The main advantages are described as follows:

- ID-based cryptosystem in ad hoc network solves the problem of traditional public key cryptosystem by eliminating the necessity for the infrastructure to authenticate public keys and manage certificates and directories to store certificates.

- We provide an efficient routing protocol with more concrete security by using ID-based aggregate signature. IDbased signature scheme eliminates the necessity to verify certificates and transfer them. And ID-based aggregate signature, which is the compression of many signatures inte only one signature, reduces the communication cost to constant in routing protocol,

The rest of the paper is organized as follows. In section II, we describe ID-based cryptosystem and propose an ID-based aggregate signature scheme. Then we present the securityenhanced model in section III. In section IV and V, we analyze our model in both security and performance aspects. Finally we conclude the paper in section VI.

\section{ID-BASED CRYPTOSYSTEM}

In this section, we explain the concept of ID-based cryptosystem and propose an $\mathrm{ID}-\mathrm{Based}$ aggregate signature firstly.

\section{A. The Concept of ID-Based Cryptosystem}

The main problem of traditional public key cryptosystem is the assumption of the infrastructure to authenticate public keys and manage certificates. To simplify them, the concept of ID-based cryplosystem was proposed by Shamir [13]. In this system, an entity's public and unique identification information such as email address or device's serial number plays the role of its public key. So the users need not to exchange their public keys and associated certificates. Another advantage is that it eliminates the need for directories to store certificates. 
Recent ID-based cryptosystems make use of the bilinear map proposed by Boneb and Franklin [1]. And the Weil and Tate pairings on elliptic curves are well known implementations of it. Let $G_{1}$ and $\mathbb{G}_{2}$ be two cyclic groups of order $q$ for some large prime $p$. A map $e: \mathbb{G}_{1} \times \mathbb{G}_{1} \rightarrow \mathbb{G}_{2}$ is said to be bilinear if it satisfies the following properties:

- Bilinear

For all $P, Q \in \mathbb{G}_{1}$ and for all $a, b \in \mathbb{Z}_{q}$, we have $e(a P, b Q)=e(P, Q)^{a b}$.

- Non-degenerate

If $P$ is a generator of $\mathbb{G}_{1}$, then $e(P, P)$ is a generator of $\mathrm{G}_{2}$.

- Computable

There is an efficient algorithm to compute $e(P, Q)$ for any $P, Q \in \mathbb{G}_{1}$.

The Bilinear Diffe-Hellman Problem (BDHP) in $<\mathbb{G}_{1}, \mathbb{G}_{2}, e>$, which is the basis of security in these systems, can be stated as follows:

- given $P, a P, b P, c P \in \mathbb{G}_{1}$ for any $a, b, c \in \mathbb{Z}_{q}$, compute $e(P, P)^{a b c}$.

\section{B. ID-Based Aggregate Signature}

The concept of aggregate signature scheme, recently introduced in [2], is to combine and compress many signatures into a short signature. Unlike multisignature schemes, it aggregates signatures on distinct messages. Another advantages are that anyone can aggregate signatures and can incrementally add its own signature into the aggregated signature. It was proved that this scheme in a Gap Diffie-Hellman group is secure against a chosen-key attack, in other words, an adversary can not forge an aggregate signature without all private keys used in. But the scheme was designed for generic Gap Diffie-Hellman group, and is not suitable for ID-based signature schemes [10], [4], [3].

Here we present an ID-based aggregate signature scheme based on the signature scheme from [3]. The security of it can be proved similarly to the scheme in [2]. This scheme consists of six algorithms, and there is a $\mathrm{KGC}$ which is trusted by all users.

\section{- Setup}

KGC chooses a generator $P$ of $\mathbb{G}_{1}$, picks a master secret key $s \in \mathbb{Z}_{q}$ and set $P_{\text {pub }}=s P$. It also select two collision resistant hash functions

$$
\begin{aligned}
& H_{1}:\{0,1\}^{*} \rightarrow \mathbb{Z}_{q} \\
& H_{2}:\{0,1\}^{*} \rightarrow \mathbb{G}_{1},
\end{aligned}
$$

The key $s$ is only known to $\mathrm{KGC}$ and the others are publicly known.
- Extract

When a signer requests the private key associated to his own identity $I D, \mathrm{KGC}$ computes

$$
D_{I D}=s H_{2}(I D)
$$

and sends it. The public key of him is set to

$$
Q_{I D}=H_{2}(I D) \text {. }
$$

\section{- Sign}

Given a private key $D_{I D}$ and a message $M \in\{0,1\}^{*}$, the signer chooses a random number $r \in \mathbb{Z}_{q}$, computes

$$
\begin{aligned}
U & =r Q_{I D} \\
h & =H_{1}(M) \\
V & =(r+h) D_{I D} .
\end{aligned}
$$

Then $\sigma=(U, V)$ becomes the signature.

- Verify

Given an identity $I D$, a message $M$ and a signature $\sigma=$ $(U, V)$, the verifier computes

$$
\begin{aligned}
Q_{I D} & =H_{2}(I D) \\
h & =H_{1}(M)
\end{aligned}
$$

and checks whether

$$
e\left(h Q_{I D}+U, P_{p u b}\right)=e(V, P) .
$$

If it is true, then the signature is valid. Otherwise, it is not valid and rejected.

\section{- Aggregate}

Given an aggregate signature $\sigma=(U, V)$ on distinct messages $M_{1}, \ldots, M_{i-1}$ and a signature $\sigma^{\prime}=\left(U^{\prime}, V^{\prime}\right)$ on a message $M_{i}$, the aggregator verify that $M_{i}$ is different from any other messages. If it is true, he computes

$$
\begin{aligned}
& U=U+U^{\prime} \in \mathbb{G}_{1} \\
& V=V+V^{\prime} \in \mathbb{G}_{1} .
\end{aligned}
$$

Then $\sigma=(U, V)$ becomes the aggregate signature on $M_{1}, \ldots, M_{i}$.

\section{- AggregateVerify}

Given identities $I D_{1}, \ldots, I D_{n}$, distinct messages $M_{1}, \ldots$, $M_{n}$, and an aggregate signature $\sigma=(U, V)$, the verifier computes

$$
h_{i}=H_{1}\left(M_{i}\right)
$$

for all $1 \leq i \leq n$. Then he checks whether

$$
e\left(\sum_{i=1}^{n} h_{i} Q_{I D_{i}}+U, \dot{P}_{p u b}\right)=e(V, P) \text {. }
$$

If it is true, all the signatures in $\sigma$ are valid. Otherwise, at least one of the signatures is invalid. 


\section{SECURITY ENHANCED MODEL}

In this section, we propose a security enhanced model to provide a comprehensive answer to the security problems in ad hoc network. We firstly adopt ID-based cryptosystem to solve the problem of traditional public key cryptosystem. Secondly, we present a secure routing solution based on ID-based aggregate signature which is proposed in section II. Using ID-based aggregate signature, our solution reduces the size of not only signature lists but also certificate chain and avoids the inappropriate assumptions for the nature of ad hoc network.

\section{A. Initialization Phase}

Initialization phase is performed only once prior to the formation of the ad hoc network. In this phase, off-line server sets up system parameters and distributes each node's private key securely.

\section{Setup System Parameters}

1) Off-line server chooses a generator $P$ of $\mathbb{G}_{1}$, picks a master secret key $s \in \mathbb{Z}_{p}$ and set $P_{p t c b}=s P$. The key $s$ is only known to off-line server and the others are publicly known.

2) It also select two cryptographic hash functions $H_{1}$ : $\{0,1\}^{*} \rightarrow \mathbb{Z}_{q}$ and $H_{2}:\{0,1\}^{*} \rightarrow \mathbb{G}_{1}$.

\section{Private Key Generation}

1) Each intial node with the identity ID must have a pair of public and private keys. Each node gets its own public key by computing $Q_{I D}=H_{2}(I D)$.

2) For each node, the server computes its private key $D_{I D}=s Q_{I D}$ with the master secret key $s$. Because $s$ is kept secret by the server, anyone else cannot compute the private key.

\section{B. Route Discovery Phase}

In this phase, we present a secure routing protocol based on on-demand routing protocols such as DSR [7] or AODV [11] where a node attempts to discover a route to some destination only when it has a packet to send to the destination. To secure a routing protocol in ad hoc network, the authentication of nodes in the network and the integrity verification of messages between nodes are required. We solve the requirements by using ID-based signature and ID-based aggregate signature. In particular, ID-based aggregate signature is used for a destination node to authenticate each intermediate node in the route.

Route discovery makes a node discover dynamically a route to any other node. Route discovery has three stages: the initiator node broadcasts a route request message called RREQ, the intermediate nodes process the RREQ message, and the target node receiving the RREQ message returns a route reply message called RREP to the initiator node. To secure the route discovery, the initiator node and the intermediate nodes generate their own signatures and integrate it into the original aggregate signature. And by verifying the aggregate signature, the target node can authenticate each intermediate node on a path and check the integrity of the message. The main advantage is that it requires less communication cost. It needs no certificate chain and the length of aggregate signature is not increased through the path.

A route request message contains six fields: $\angle R R E Q$, initiator, target, seq, nodelist, aggsign $>$. The $R R E Q$ is a packet type identifier, the initiator is the identity of the initiator node, and the target is the identity of the target node. The seq is incremented whenever the initiator issues a new RREQ, the nodelist is a list of intermediate nodes on the route between initiator and target nodes, and the aggsign is a aggregate signature integrated by initiator and intermediate nodes.

When any node receives a RREQ, it processes the message according to the following steps:

1) If the RREQ message from the initiator node has received recently, namely the pair (initiator, seq) for the RREQ is found in this node's received request list, then discard the message and do not process it further.

2) Otherwise, if this node is not the target of the RREQ, then add this node's identity to the nodelist and generate its own signature on the following fields: $\angle R R E Q$, initiator, target, seq, nodelist>. And aggregate its signature into the aggregate signature, aggsign, then re-broadcast the message. The length of aggregate signature does not change, so it does not require any communication overhead.

3) Otherwise, namely if this node is the target of the RREQ, then verify the aggregate signature, aggsign, in the RREQ.

a) If the aggregate signature is valid, then return a RREP message to the initiator node.

b) Otherwise, discard the message and do not process it further.

A route reply message contains the following fields: $<R R E P$, iarget, initiator, seq, nodelist, sign $>$. The RREP is a packet type identifier and the target, initiator, nodelist fields are set to the corresponding values from the RREQ message. The seq is incremented whenever the target node issues a new RREP and the sign is a signature of the target node.

To describe this procedure in detail, we take an example that the initiator node $A$ attempts to discover a route to the target node $D$. Let node $A$ 's next hop be node $B, B$ 's next hop be node $C$, and $C$ 's next hop be the target node $D$.

$$
\begin{aligned}
A \rightarrow \text { all }: & {\left[R R E Q, I D_{A}, I D_{D}, \text { seq, }(0]\right. \text { AggSign }} \\
& A g g \text { Sign }= \\
& {\left[R R E Q, I D_{A}, I D_{D}, \text { seq, }(0] \text { Sign }_{A}\right.} \\
B \rightarrow \text { all }: \quad & {\left[R R E Q, I D_{A}, I D_{D}, \text { seq, }(B)\right] \text { AggSign } }
\end{aligned}
$$




$$
\begin{aligned}
& \text { AggSign }=\text { AggSign }+ \\
& {\left[R R E Q, I D_{A}, I D_{D}, \text { seq, }(B)\right] \operatorname{Sign}_{B}} \\
& C \rightarrow \text { all : }\left[R R E Q, I D_{A}, I D_{D}, \operatorname{seq},(B, C)\right] \text { AggSign } \\
& \text { AggSign }=\text { AggSign }+ \\
& {\left[R R E Q, I D_{A}, I D_{D}, \operatorname{seq},(B, C)\right] \operatorname{Sign}_{C}} \\
& D \text { : Verify the aggregate signature } \\
& A g g \operatorname{Sign} \stackrel{?}{=} \operatorname{Sign}_{A}+\operatorname{Sign}_{B}+\operatorname{Sign}_{C} \\
& D \rightarrow A \quad: \quad\left[R R E P, I D_{D}, I D_{A}, s e q^{\prime},(B, C)\right] \operatorname{Sign}_{D}
\end{aligned}
$$

\section{Route Maintenance Phase}

In on-demand routing protocols, there are no periodic routing update messages. Thus, while a route is in use, intermediate nodes notify the sender of any routing error. This procedure is called route maintenance. Assume that for a route between source node $A$ and destination node $D$, a intermediate node $B$ comes to detect that a link in the route is broken due to some reasons like node's mobility or unreliability of ad hoc network. Then node $B$ sends node $A$ a route error message called RERR as follows:

$$
B \rightarrow A \quad: \quad\left[R E R R, I D_{A}, I D_{D}, \text { seq }\right] S_{i g n}
$$

The $R E R R$ is a packet type identifier, the $I D_{A}$ is the identity of the source node $A$, and the $I D_{D}$ is the identity of the destination node $D$. The seq ensures the RERR message is fresh and the Sign $_{B}$, node $B$ 's signature, guarantees not only the integrity of the RERR message but also the non-repudiation of node $B$.

\section{SECURITY ANALYSIS}

In ad hoc network, several properties for security are required. Among them, authentication, integrity, and secure routing are more important because they are the bases of other properties.

- Authentication : A node wants to be sure that it is talking to whomever it intends.

- Secure Routing : A node wants to find a correct and reliable route path.

- Integrity : A node wants to be sure that the message cannot be altered in an unauthorized way.

In this section, we analyze and discuss our model in the aspect of each category.

\section{A. Authentication}

Authentication is a basic building block of security. But the traditional mechanisms including Public Key Infrastructure(PKI) require pre-established infrastructure to manage certificates. It must be assumed that there exists a trusted authority through the whole lifetime of the network to issue new certificates, revoke compromised certificates, and renew expired certificates. However this assumption is not suitable due to the nature of ad hoc network.

On the other hand, our model is based on ID-based cryptosystem. Its main advantage is that it eliminates the necessity to manage certificates because the unique identity of a node is used as the public key of itself. Still the problem of revocation remains. But in many cases the compromised node has a critical vulnerability so it is recommended. not to use it anymore.

\section{B. Secure Routing}

Secure routing is a special requirement in ad hoc network because routing functions are carried out by all available nodes. Current secure routing protocols take into account active attacks performed to disrupt the correct route functioning by modifying route information, fabricating false route information, and impersonating other nodes.

The weak point of ARAN is that there is no way to prevent a malicious intermediate node from modifying route path because only one-hop neighbor node is authenticated and there is no mechanism to check the integrity of the route. An intuitive solution is that the intermediate node adds its ID in the path list and also adds its signature on the modified path list without removing other signatures. But this solution increases the number of signatures and certificates added to the original message proportionally to hop count, and thus requires a heavy communication cost.

Our model solves this problem with aggregate signature. Because aggregate signature merges all signatures through the path into a single one and no certificates are added, our model efficiently defends against not only incorrect modification of path but also impersonating. And the node fabricating false route information can be excluded later because ID-based signature provides non-repudiation. In the aspect of computation cost, our model has a similar cost to ARAN. Because if the length of route path is $n$, the cost of verifying an aggregate signature is similar to the cost of verifying $n$ signatures.

\section{Integrity}

Integrity can be guaranteed by adding ID-based signature to the message. Because only the node that holds the identity and associated private key can generate a valid signature, any other node can not alter the message. 


\section{PERFORMANCE ANALYSIS}

In this section, we compare our model with previous models. We only consider the communication cost of RREQ message when the length of route path is $n$, because this type of message has a remarkable contrast. In the previous models, suppose that 1024 bits RSA key is used, the size of certificate is 900 bytes, and the size of signature is 150 bytes. Then the total number of bytes to be transferred additionally is

$$
\sum_{i=1}^{n}(900+150) i=525 n(n+1) .
$$

On the other hands, suppose that our model uses 160 bits key in the elliptic curve system having similar security level to 1024 bits RSA key, and the size of signature is 62 bytes. Then the total number of bytes to be transferred additionally is only $62 n$ because certificates are not used and aggregate signature is used. Therefore our model reduces communication cost significantly.

In the aspect of computation cost, our model performs one verification of aggregate signature which is the same as verification of $n$ signatures. But there is no need to verify any certificate. Therefore, our model is more efficient than others.

\section{CONCLUSION}

Currently the major issues in ad hoc network are secure routing, authentication, and key management. Among them, key management itself depends very closely on authentication and sound key management is not possible without sound authentication.

In this paper, we proposed a security-enhanced model with ID-based cryptosystem. Our model provides a sound authentication mechanism without certificate management problem. Also we proposed a secure routing protocol with aggregate signature, which reduces communication cost significantly. We expect that our model contributes to designing a securityenhanced environment appropriate to ad hoc network.

\section{REFERENCES}

[1] D. Boneh and M. K. Franklin. Identity-based encryption from the weil pairing. In Proceedings of the 21st Anmual Intermational Cryptology Comference on Advances in Cryptology, pages 213-229. Springer-Verlag, 2001 .

[2] D. Boneh, C. Gentry, B. Lynn, and H. Shacham. Aggregate and verifably eacrypted signature from bilinear maps. In Proceedings of Advances in Cyptology. EUROCRYPT 2003, pages 416-432. SpringerVerlag, 2003.

[3] J. C. Cha and J. H. Cheon. An identity-based signature from gap diffiehellman groups. In Practice and Theory in Public Key Cryptography PKC'2003, pages 18-30. Springer-Verlag, 2003.

[4] F. Hess. Efficient identicy based signature schemes based on pairings. In Selected Areas in Cryprography - SAC'2002, pages 310-324. SpringerVerlag, 2003.

[S] Y. Hu, D. B. Johnson, and A. Perrig. Sead: Secure efficient distance vector routing for mobile wireless ad hoc networks. In Praceedings of the Fourth IEEE Workshop on Mobile Computing Systems and Applications, page 3. IEEE Computer Society. 2002.

[6] Y. Hu. A. Perrig, and D. B. Johnson. Ariadne:- a secure on-demand routing protocol for ad hoc networks. In Proceedings of the 8th annual intemational conference on Mobile computing and networking, pages 12-23. ACM Press, 2002.

[7] D. Johson. Routing in ad hoc networks of mobile hosts. In Proceedings of the IEEE Workshop on Mobile Computing Sysiems and Applications, pages 158-163, 1994.

[8] R. Molva and P. Michiardi. Security in ad hoc networks. In Proceedings of Personal Wireless Communications (PWC'03), pages 736-775, 2003.

[9] P. Papdimitratos and Z. Haas. Secure routing for mobile ad hoc networks. In Proceedings of the SCS Communication Networks and Distributed Systems Modeling and Simulation Comverence(CNDS 02), 2002.

[10] K. G. Paterson. Id-based signatures from pairings on elliptic curves. Electronics Letters, 38(18):1025-1026, 2002.

[11] C. E. Perkins and E. M. Royer. Ad-hoc on-demand distance vector routing. In Praceedings of the 2nd IEEE 2003 Workshop on Mobile Compuing Systems and Applications, pages 90-100, 1999.

[12] K. Sanzgiri, B. Dahill, B. N. Levine, C. Shields, and E. M. BeldingRoyer. A secure routing protocol for ad hoc networks. In Proceedings of the 10th IEEE Conference on Network Protocols (ICNP'02), pages 78-89. IEEE Computer Society, 2002.

[13] A. Shamir. Identity-based cryptosystems and signature schemes. In Proceedings of CRYPTO 84 on Advances in cryptology, pages 47-53. Springer-Verlag Now York, Inc., 1985

[14] S. Yi and R. Kravets. Moca: Mobile certificate authority for wireless ad hoc networks. In Proceedings of the 2nd Anmual PKI Research Workshop (PKI'03), 2002. 